\title{
Endovascular repair of a type B aortic dissection with a right-sided aortic arch: case report
}

\author{
Weimin Zhou
}

\begin{abstract}
Right-sided aortic arch is a rare anomaly, and aortic dissection involving a right-sided aortic arch is extremely rare. We report the case of a 65-year-old man with a right-sided aortic arch and a right descending aortic dissection and a stent-graft was accurately deployed without perioperative complications. There were no any complaints and complications after 18 months follow-up. The CTA demonstrated that the false lumen was largely thrombosed only with a mild type II endoleak and a mild descending aortic expansion. We feel that endovascular repair is feasible to patient of type B aortic dissection with a right-sided aortic arch. However, long-term clinical efficacy and safety have yet to be confirmed.
\end{abstract}

Keywords: Aortic dissection, Right-sided aortic arch, Stent-graft

\section{Background}

Right-sided aortic arch is a rare anomaly, and aortic dissection involving a right-sided aortic arch is extremely rare. To our knowledge, previous reports that we have identified in English literature include only fifteen cases of aortic dissection involving a right-sided aortic arch [1] (through a Medline database search from 1966 to October 2011 and the search strategy "right-sided aortic arch and aortic dissection") and they were performed with traditional surgical procedures except for one report has described endovascular treatment [2]. We are presenting a case of type B aortic dissection associated with a rightsided aortic arch and aberrant left subclavian artery, in which a successful endovascular stent-graft placement was performed.

\section{Case presentation}

A 65-year-old man was admitted to our institution with acute onset chest and back pain and computer tomographic angiography (CTA) demonstrated a right-sided aortic arch; a right descending aortic dissection with an aberrant left subclavian artery (LSA) arising from Kommerell's diverticulum (Figure 1). The size of descending aortic is $52 \mathrm{~mm}$.It also defined the ordering of the aortic arch branches as left common carotid, right common carotid, right subclavian,

Correspondence: drzwm@sina.com

Department of Vascular Surgery, the second affiliated hospital of Nanchang University, Nanchang, China and left subclavian arteries from proximal to distal; a celiac axis, superior mesenteric artery and right renal artery originating from true lumen and left renal artery originating from false lumen and a little pleural fluid in bilateralis thoracic cavity.

The procedure was performed under general endotracheal anesthesia and full hemodynamic monitoring one day later. The right femoral artery was exposed and cannulated with a 6-French catheter sheath, from which an angiographic mark catheter was put into the aortic true lumen via a 0.035 inch guidewire. Digital subtraction angiography (DSA) showed a right-sided aortic arch, type $\mathrm{B}$ aortic dissection, and dominanted right vertebral artery (Figure 2). We locked the "C" arm in right anterior oblique (RAO) $45^{\circ}$ to unfold the aortic arch, and cannulated with a 21-French delivery introducer sheath via a 0.035 inch Lunderquist super stiff guidewire through the right femoral artery. A stent-graft (Talent, 4242150, Medtronic, USA) was accurately deployed. Completion angiogram revealed no evidence of type I endoleak, sealing of the false lumen, no visualization of left subclavian artery and no visceral vessels malperfused (Figure 3). His postoperative course was uneventful and discharged postoperative day 4. No complaint and complication was documented during 18 months follow-up. The CTA demonstrated that the false lumen was largely thrombosed only with a mild type II endoleak and a 


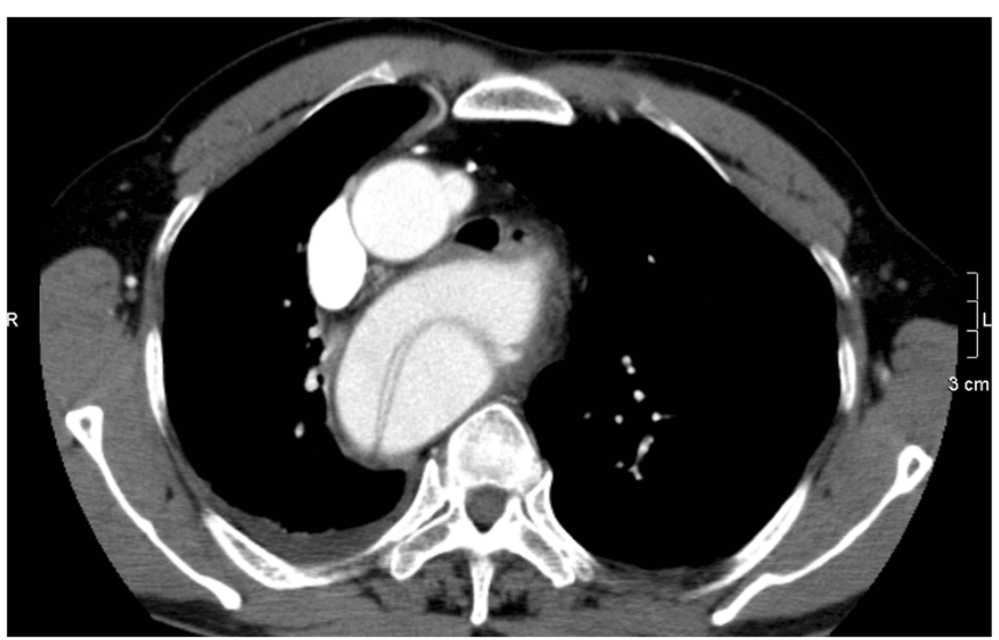

Figure 1 CTA demonstrated a right-sided aortic arch and a right descending thoracic aorta, a type B aortic.

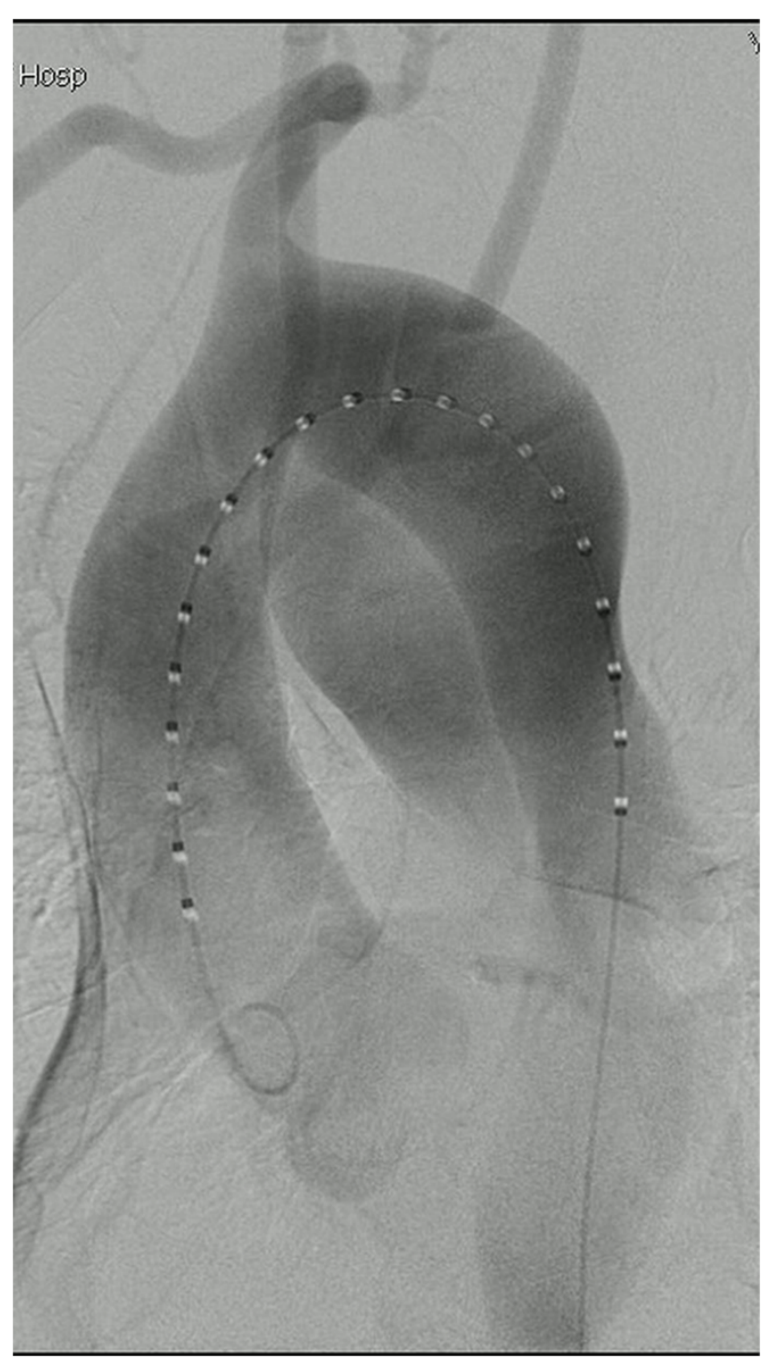

Figure 2 (See legend on next page.) 
(See figure on previous page.)

Figure 2 Digital subtraction angiography showed a right-sided aortic arch and the right vertebral artery is dominant. A LAO projection showed the ordering of the aortic arch branches as left common carotid, right common carotid, right subclavian and left subclavian arteries from proximal to distal.

mild descending aortic expansion (The size of descending aortic is 66mm) (Figure 4).

\section{Discussion}

Right-sided aortic arch occurs in approximately $0.1 \%$ of the population. There are three major types [1]. Type I, with mirror-imaged branching of the major arteries. Type II, with aberrant subclavian artery. The ordering of the aortic arch branches as left common carotid, right common carotid, right subclavian, and LSA arising from Kommerell's diverticulum from proximal to distal. Type III, with isolation of the subclavian artery. Type I

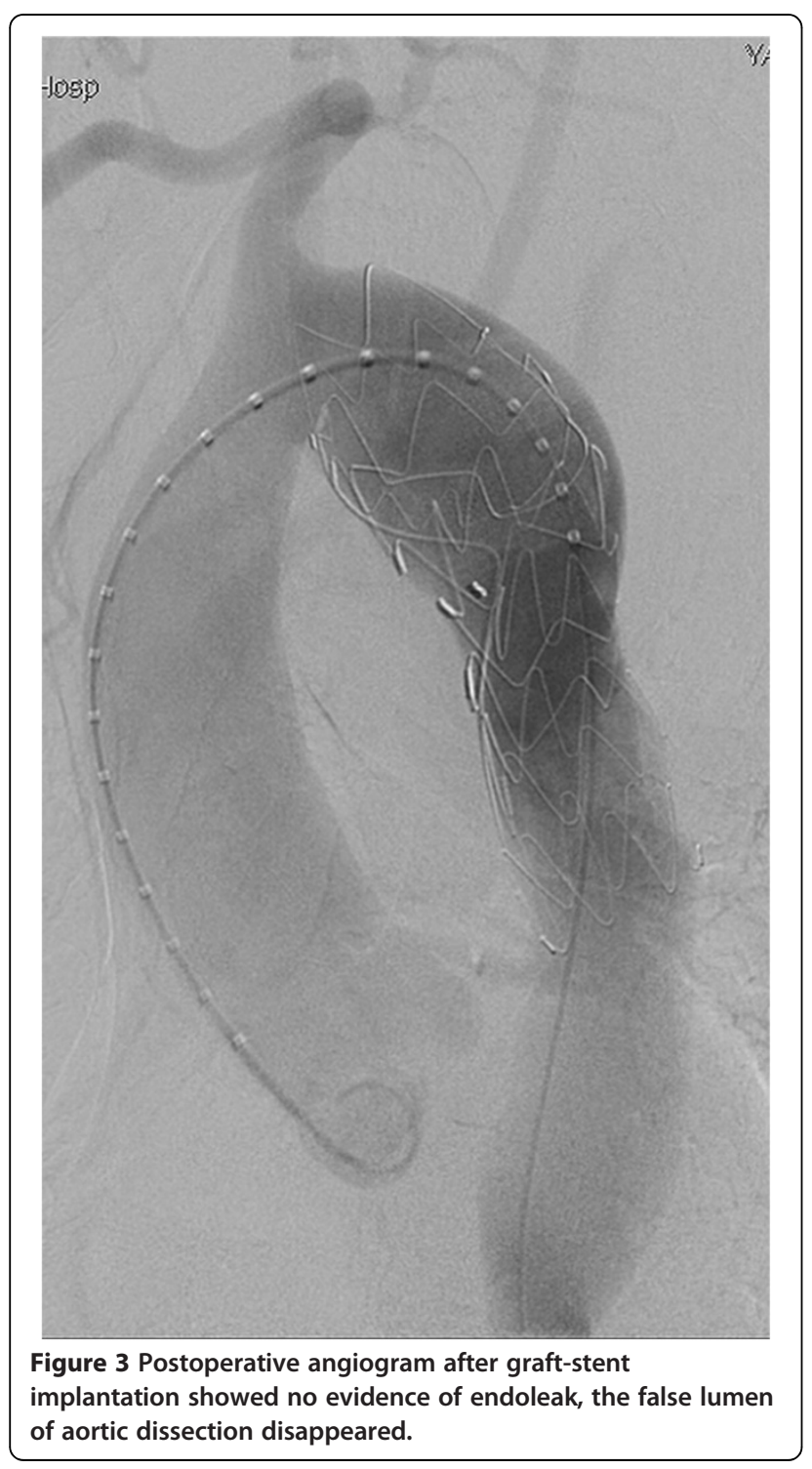

represent 59\% of all right aortic arches, type II $39.5 \%$, and type III $0.8 \%$.

In 1994, Dake and colleages [3] first reported successful transluminal placement of endovascular stent-grafts for the treatment of aortic dissection, and showed good early results. This technique is less invasive than traditional surgery with acceptable morbidity and mortality rates. Endovascular treatment of right-sided aortic arch aneurysms is appealing because it avoids a thoracotomy or median sternotomy, and it obviates the need for circulatory arrest, which is commonly required with the conventional approach. Okada and associates [4] demonstrated the feasibility of endovascular repair in a 76-year-old man with an aneurysm that involved a rightsided aortic arch. Naoum and colleagues [5] described the successful surgical repair in a 58-year-old man of descending thoracic aortic aneurysm with a right-sided aortic arch by the combined use of a left carotid to subclavian artery bypass followed by endovascular stentgraft placement. Yamazaki [2] first reported a successful stent-graft treatment in a 56-year-old man suffered from type $\mathrm{B}$ aortic dissection involving a right aortic arch. In the present case, the anatomic features made the landing zone between the right subclavian artery and the thoracic aortic intimal tear long enough to accommodate a stent-graft without causing basal artery ischemia. And let the "C" arm in right anterior oblique (RAO) $45^{\circ}$ other than left anterior oblique (LAO) to unfold the aortic arch intraoperatively. After 18 months follow-up, there is no dizzy, no swoon, no left upper extremity necrosis, no extremities myodynamia degrade, no nervous system complications, and no dysphagia stridor. However, a mild type II endoleak and a mild descending aortic expansion most probably come from the LSA back-flow.

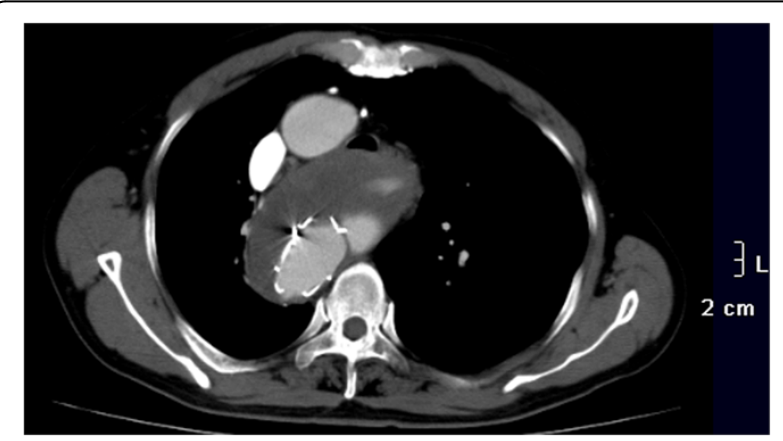

Figure 4 After 18 months follow-up, CTA documented that the false lumen was largely thrombosed only with a mild type II endoleak and a mild descending aortic expansion. 
We are planning to followup of the type II endoleak and aortic expansion, and ligate or embolize the LSA when necessary.

\section{Conclusion}

Although early results are promising, long-term clinical efficacy and safety have yet to be confirmed.

\section{Consent}

Written informed consent was obtained from the patient for publication of this Case report and any accompanying images. A copy of the written consent is available for review by the Editor-in-Chief of this journal.

\section{Competing interests}

The author declare that he has no competing interests.

\section{Acknowledgements}

The author thanks Jiang Junhao,MD for retouching the manuscript.

Received: 25 January 2012 Accepted: 21 January 2013

Published: 23 January 2013

\section{References}

1. Cinà CS, Arena GO, Bruin G, Clase CM: Kommerell's diverticulum and aneurysmal right-sided aortic arch: a case report and review of the literature. J Vasc Surg 2000, 32:1208-1214.

2. Yamazaki I, Imoto K, Ichkawa $Y$, et al: Stent-graft treatment of type B aortic dissection involving the right aortic arch (case report). Jpn Circ J 2000, 64:727-728

3. Dake MD, Miller MC, Semba CP, Mitchell RS, Walker PJ, Liddell RP: Transluminal placement of endovascular stent-grafts for the treatment of descending thoracic aortic aneurysms. N Engl J Med 1994, 331 (26):1729-1734.

4. Okada K, Sueda T, Orihashi K, Watari M, Naito A: Endovascular stent-graft repair for thoracic aortic aneurysm associated with right-sided aortic arch. J Thorac Cardiovasc Surg 2001, 122:185-186.

5. Naoum JJ, Parenti JL, LeMaire SA, Coselli JS: Endovascular repair of a rightsided descending thoracic aortic aneurysm with a right-sided aortic arch and aberrant left subclavian artery. Ann Thorac Surg 2008, 85:1074-1076.

\section{doi:10.1186/1749-8090-8-18}

Cite this article as: Zhou: Endovascular repair of a type B aortic dissection with a right-sided aortic arch: case report. Journal of Cardiothoracic Surgery 2013 8:18.

\section{Submit your next manuscript to BioMed Central and take full advantage of:}

- Convenient online submission

- Thorough peer review

- No space constraints or color figure charges

- Immediate publication on acceptance

- Inclusion in PubMed, CAS, Scopus and Google Scholar

- Research which is freely available for redistribution 